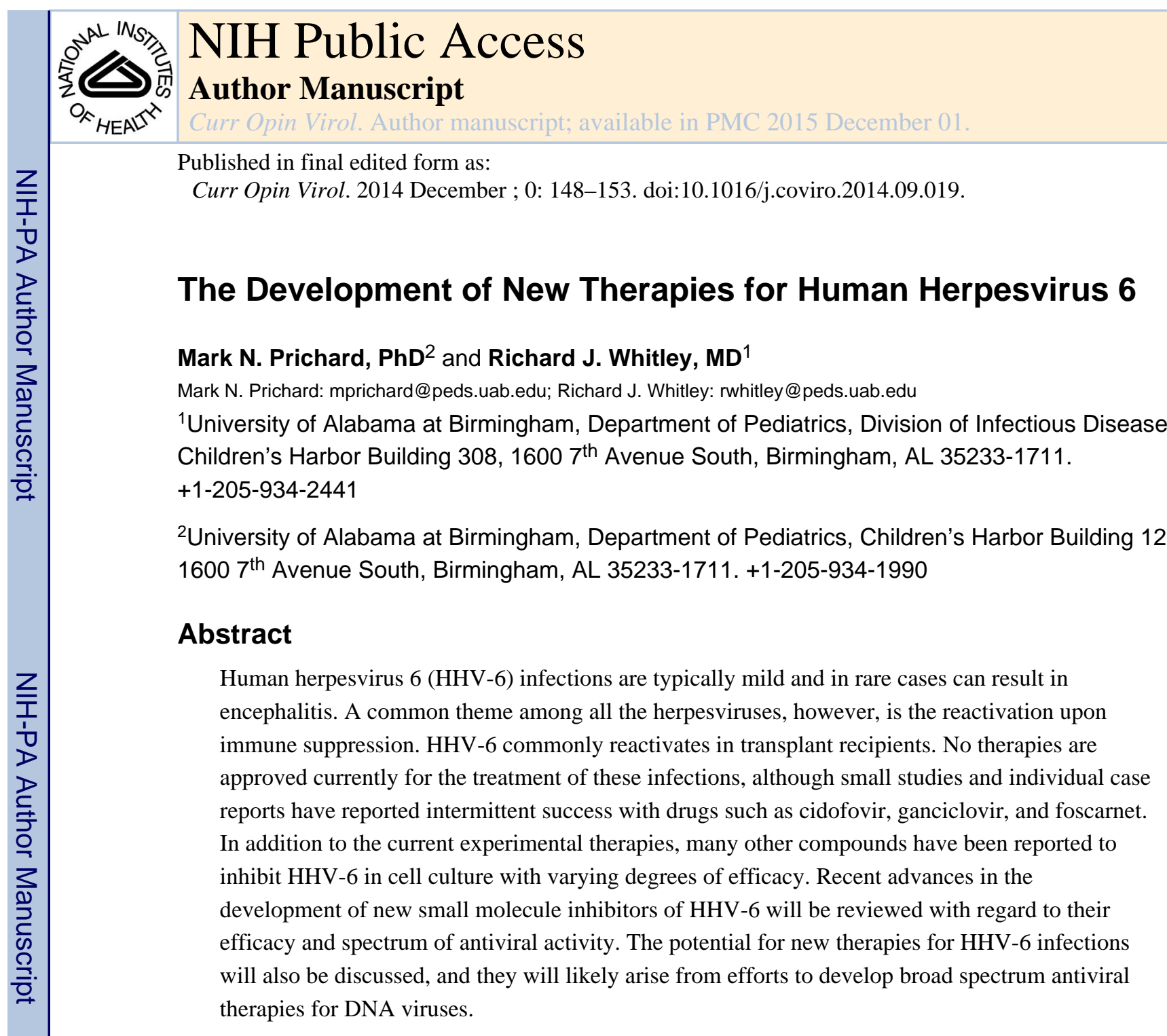

\title{
Introduction
}

Human herpesvirus 6 A (HHV-6A) and human herpesvirus 6 B (HHV-6B) are members of the betaherpesvirus subfamily, as is cytomegalovirus (CMV) and Human Herpesvirus 7 (HHV-7). Primary infections with the Roseoloviruses HHV-6A and HHV-6B typically occur early in life with HHV-6B being the most common [1]. The two HHV-6 viruses are distinct entities and are classified as different species [2,3]; they are associated with different clinical manifestations, yet it is not always feasible to distinguish the viruses in clinical studies so data from viruses are generally analyzed together and reported simply as HHV-6 infections. Here, we will specify the specific virus where possible and will simply use the HHV-6 designation where it is not. Primary infection with HHV-6B has been shown to be the cause of exanthem subitum (roseola) in infants [4], and can also result in an

(C) 2014 Elsevier B.V. All rights reserved.

Correspondence to: Richard J. Whitley, rwhit ley@peds . uab .edu.

Publisher's Disclaimer: This is a PDF file of an unedited manuscript that has been accepted for publication. As a service to our customers we are providing this early version of the manuscript. The manuscript will undergo copyediting, typesetting, and review of the resulting proof before it is published in its final citable form. Please note that during the production process errors may be discovered which could affect the content, and all legal disclaimers that apply to the journal pertain. 
infectious mononucleosis-like illness in adults [5]. Infections caused by HHV-6A and HHV-7 have not been well characterized and are typically reported in the transplant setting $[6,7]$. Serologic studies indicated that most people become infected with HHV-6 by the age of two, most likely through saliva transmission [8]. The receptors for HHV-6A and HHV-6B have been identified as CD46 and CD134, respectively $[9,10]$. This facilitates entry into many cell types including CD4+ cells, CD8+ T-cells, natural killer cells, monocytes, epithelial cells and brain-derived cells [11].

The development of therapies for HHV-6 infections has been limited because of the lack of unequivocal association between infection and disease that warrants intervention. Roseola infections are typically mild and do not warrant therapy. Other infections caused by HHV-6 have been incriminated in a variety of human illnesses; however, the lack of cause and effect has impeded drug development and controlled studies of existing medications in order to establish value of treatment are wanting. In large part, such associations may be dependent upon controlled clinical trials that establish the value of therapies in targeted diseases. Diseases associated with HHV-6 infection have included encephalitis and infections in immunocompromised host, particularly interstitial pneumonitis [12]. In addition, infection has been incriminated as a cause of multiple sclerosis, as has been the case for numerous other viral agents [13]. Reactivation of HHV-6 frequently occurs during immune suppression and is seen in 50\% of all bone marrow and 20-30\% of solid organ transplant recipients $[7,14]$. Two clinical studies suggest a role of HHV-6 in contributing to morbidity in hematopoietic stem cell transplant recipients. Specifically the early reactivation with increasing viral load was associated with fever, skin rash, diarrhea, pulmonary complications and neurologic disorders [15]. A second study utilized prophylactic ganciclovir in a placebo-controlled study that demonstrated drug administration decreased the probability of skin rash, interstitial pneumonitis, diarrhea and thrombatic microangiopathy (TMA) [16]. Diagnostic procedures and sequencing analyses have shown that the viral genome can integrate within telomeric regions of chromosomes in some individuals, although its significance remains unclear $[17,18]$. While these studies do not define disease etiology, they provide potential indications for the development of therapeutics for antiviral agents.

\section{Methodologic Assays}

Several methodologies have been employed to detect activity of small molecules against all of the betaherpesviruses and will be summarized specifically for HHV-6 infection. All isolates of HHV-6A and HHV-6B replicate well in phytohemagglutinin-stimulated umbilical cord blood lymphocytes and exhibit a prolonged replication cycle characteristic of this subfamily [19]. Additional cell lines that support viral replication have been identified and are generally used in the evaluation of antiviral activity. The first report of antiviral activity against GS strain of HHV-6A was described in a T-lymphoblastoid cell line (HSB-2) [20]. The Z29 strain of HHV-6B replicates well in Molt-3 cells, and this line is most often used in evaluating the efficacy of antiviral agents [21]. In all cells, viral replication can be assessed by DNA hybridization, quantitative PCR, and flow cytometry, but cytopathology is also apparent in some cell lines [20,22, 23]. 


\section{Molecules with Antiviral Activity Against HHV-6}

The susceptibility of HHV-6 to antiviral drugs seems to be distinct from that of CMV, although CDV, PFA, and GCV all appear to inhibit virus replication in vitro with modest efficacy $[20,24]$. To summarize the activity of the more commonly used compounds, the in vitro efficacy from several manuscripts is shown in Table 1. Additional effective agents are in various stages of development and the most promising small molecules will be discussed in detail below (Fig. 1). Immunotherapeutic strategies have also been reported for the therapy of HHV-6 infections but are outside the scope of this review [25].

\section{Ganciclovir}

The one molecule studied extensively for therapy of putative HHV-6 infection is ganciclovir, a nucleoside analog that was synthesized in the 1980s for the treatment of CMV infection. This compound is phosphorylated by the U69 kinase in HHV-6 and the active triphosphate metabolite inhibits the DNA polymerase. The activity of ganciclovir in vitro is dependent upon the assay that has been employed. Utilizing an immunofluorescence assay, ganciclovir was reported to have a minimal level of in vitro activity at $>25 \mu \mathrm{M}$ [20]. However, in a more sensitive assay that utilized cord blood lymphocytes, Yoshida and colleagues reported activity in the $\mu \mathrm{M}$ range [26]. In large part, this limited activity against both viruses may be related to the low level of phosphorylation by the U69 kinase, and, as a consequence, the reduced inhibition of DNA polymerase by the active metabolite [27, 28]. Resistance to the drug maps both the U69 protein kinase as well as the U38 DNA polymerase, and the mechanism of action is thought to be similar to that against CMV [29, 30].

Several clinical trials have suggested the value of ganciclovir, but from a very limited perspective [16,31-37]. Of note, resistant virus has been detected in a number of transplant patients and is not unexpected given the modest efficacy of ganciclovir against this virus $[38,39]$.

With the development of the oral formulation of ganciclovir, valganciclovir, an alternative to intravenous therapy exists and provides an opportunity for the performance of controlled clinical trials with greater ease of drug administration in those populations that tolerate orally administered medications.

\section{Foscarnet}

Foscarnet is employed to treat CMV infections in the immunocompromised host, particularly in the presence of antiviral resistance to ganciclovir. This drug directly binds the pyrophosphate binding site in the DNA polymerase and inhibits the activity of this enzyme. It was among the first drugs identified to have activity against HHV-6 replication in vitro at a level of approximately $25-50 \mu \mathrm{M}$, and it is active against both viruses [20, 40]. In clinical studies the drug has been used alone and with other licensed drugs, including ganciclovir and cidofovir for the treatment of HHV-6 infections [33, 35, 37, 41, 42]. Notably, the electrolyte imbalances resulting from therapy result in renal toxicity that is a deterrent to its 
use. As would be anticipated from its mechanism of action, resistance to foscarnet maps to the DNA polymerase [43].

\section{Cidofovir}

Cidofovir is licensed for the therapy of CMV infections, particularly in high-risk immunocompromised hosts. Historically, the medication was used as an alternative treatment to ganciclovir in AIDS patients with retinitis. This acyclic nucleoside phosphonate analog is phosphorylated by cellular kinases to the diphosphate and is incorporated into viral DNA by the viral DNA polymerase. With improved therapy of HIV infection, the incidence of CMV retinitis is low. Cidofovir and cyclic cidofovir inhibit the replication of both HHV-6A and HHV-6B with $\mathrm{EC}_{50}$ values of 3-9 $\mu \mathrm{M}[20,22]$. There are anecdotal reports suggesting that it may have some utility in the treatment of HHV-6 infections either alone or with other compounds and is plausible given this drug is active against all the human herpesviruses $[33,42,44]$. Drug resistance has been generated in the laboratory and maps to the HHV-6 DNA polymerase, namely the U38 gene [45].

\section{Brincidofovir}

The lipophilic derivative of cidofovir, brincidofovir or CMX001, is the hexadecyloxypropylcidofovir molecule [46]. It is a lipophilic prodrug of cidofovir that is highly active against many human DNA viruses, including HHV-6 and its mechanism of action is similar to that of CDV. It is among the most active molecules that have been tested against this virus with $\mathrm{EC}_{50}$ values of 3 and $7 \mathrm{nM}$ for HHV-6A and HHV-6B, respectively [47]. This molecule has been studied extensively in animal models against a variety of DNA viral infections, and has superior activity to cidofovir [48, 49]; however, since there is no animal model of HHV-6 infection, no similar data are currently available. Furthermore, the drug was shown to suppress CMV disease in hematopoietic cell transplant in a phase 2 clinical trial, and a pivotal phase 3 clinical trial is underway in hematopoietic stem cell transplant recipients [50]. Since the betaherpesviruses CMV and HHV-6 both exhibit similar levels of susceptibility to brincidofovir, the drug might be expected to suppress disease from both viruses in this high risk population.

\section{CMV423}

CMV423 is a novel molecule with good activity against $\mathrm{HHV}-6$ ( $\mathrm{EC}_{50}$ approximately 50 $\mathrm{nM}$ ) [51]. It inhibits a cellular protein tyrosine kinase that plays a critical role in HHV-6 viral replication [52]. Since the compound inhibits a cellular protein, the likelihood of advancement into clinical trials is unclear because of potential host cell toxicity.

\section{Cyclopropavir and Other Methylenecyclopropane Analogs}

Cyclopropavir is a methylenecyclopropane analog that is a potent inhibitor of CMV and is also active in vitro against $\mathrm{HHV}-6$ infection with $\mathrm{EC}_{50}$ values of 1 and $6 \mu \mathrm{M}$ for HHV-6A and $6 \mathrm{~B}[22,53]$. Its mechanism of action is similar to ganciclovir in that it is phosphorylated by the CMV UL97 kinase, but it also interferes with the normal function of the UL97 kinase that is critical to the replication of CMV [54-57]. In HHV-6 the UL97 homolog, U69, 
phosphorylates the drug thus, its mechanism of action appears to parallel that of ganciclovir although with superior efficacy against HHV-6 [58].

Recently, similar methylenecyclopropane analogs have been synthesized with ether and thioether substitutions at the 6 position of the purine and these analogs have superior activity to cyclopropavir [22]. These are also phosphorylated directly by the U69 kinase [58]. The mechanism of action of this series of compounds is more complex than that of cyclopropavir because of the modification of the guanine as well as the absence of a $3^{\prime}$ hydroxyl, which implies that it is likely an obligate chain terminator (Fig. 1).

\section{Benzimidazole Derivatives}

Several benzimidazole analogs have been reported to have antiviral activity against the human herpesviruses; maribavir is an L benzimidazole and is a potent inhibitor of CMV replication with submicromolar efficacy [59]. The molecule inhibits CMV UL97 kinase and is the most specific protein kinase inhibitor that has been identified to date [57].

Unfortunately, Phase III clinical trials for the prevention of CMV infection in hematopoietic stem cell transplant recipients failed to demonstrate efficacy and its further development is in doubt. This molecule also inhibits the U69 protein kinase in HHV-6, however its antiviral activity against HHV-6A and HHV-6B in cell culture is very limited as compared to CMV [60].

Another D benzimidazole analog, BDCRB (2-bromo-5,6-dichloro-1-beta-Dribofuranosylbenzimidazole), has a completely different mechanism of action and is the first described inhibitor of the CMV terminase [61]. While this molecule exhibits limited antiviral activity against HHV-6, the L analog of BDCRB is a potent inhibitor of HHV-6 with $\mathrm{EC}_{50}$ values of 2.8 and $9.7 \mu \mathrm{M}$ for HHV-6A and HHV-6B, respectively [60]. It is unknown whether this compound targets the U69 kinase, terminase complex, or other essential function, but the distinct structure activity relationship of this series of compounds against HHV-6 is clearly different from that of CMV and thus this series of compounds holds promise.

\section{Other Molecules with Activity Against HHV-6 In Vitro}

Artesunate molecules are licensed and have efficacy against malaria and, to a much more limited extent, CMV infection. The precise mechanism of action against the DNA viruses is unknown and may not be specific. For CMV infection, the $\mathrm{EC}_{50}$ is approximately $5.8 \mu \mathrm{M}$ $[62,63]$. Anecdotal reports have documented apparent efficacy in a very few cases but clinical trials will be required to assess the potential utility of this compound $[64,65]$. Efficacy has also been reported against HHV-6A with and $\mathrm{EC}_{50}$ value of $3.8 \mu \mathrm{M}$ [66]. A single report described the use of the drug in the treatment of a child with HHV-6B myocarditis, yet it was unclear that the artesunate therapy was related to the recovery of the patient [67].

Numerous other molecules have been reported to exhibit antiviral activity against HHV-6 in cell culture. 3-Deaza-HPMPA is active in the low $\mu \mathrm{M}$ range [20], however, because of toxicity it will not be advanced into clinical trials. Arylsulfone derivatives also have been

Curr Opin Virol. Author manuscript; available in PMC 2015 December 01. 
reported to exhibit activity with $\mathrm{CMV} \mathrm{EC}_{50}$ values in the at the low micromolar level and this series warrants further investigation [68]. Continued evaluation of new molecular entities will likely be required to identify potent new molecules with novel molecular targets.

\section{Conclusions}

At present, it is highly unlikely that any drug will be developed specifically for the treatment of HHV-6 infections. The availability of molecules that inhibit HHV-6 will in all probability result from spin-offs of those drugs synthesized to improved therapy of CMV infections of humans. More importantly, it underscores the need for safe and effective broad spectrum antiviral drugs that can prevent disease in high risk populations not only from CMV and HHV-6, but also from other DNA viruses such as HHV-7, herpes simplex virus, EpsteinBarr virus, varicella-zoster virus, BK virus, and adenovirus. As long as the causative role of HHV-6 in diseases with significant impact or morbidity is not established, the development of specific therapeutics for this virus will remain a relatively low priority. The focus on antiviral agents with a broad spectrum antiviral activity that includes the roseoloviruses currently has the greatest potential to yield effective therapies for these infections.

\section{References and Recommended Reading}

Papers of particular interest, published within the period of review have been highlighted as:

* of special interest

** of outstanding interest

1. Zerr DM, et al. A population-based study of primary human herpesvirus 6 infection. N Engl J Med. 2005; 352(8):768-76. [PubMed: 15728809]

2. Dominguez G, et al. Human herpesvirus 6B genome sequence: coding content and comparison with human herpesvirus 6A. J Virol. 1999; 73(10):8040-52. [PubMed: 10482553]

3. Inoue N, Dambaugh TR, Pellett PE. Molecular biology of human herpesviruses 6A and 6B. Infect Agents Dis. 1993; 2(6):343-60. [PubMed: 8012736]

4. Yamanishi K, et al. Identification of human herpesvirus-6 as a causal agent for exanthem subitum. Lancet. 1988; 1(8594):1065-7. [PubMed: 2896909]

5. Steeper TA, et al. The spectrum of clinical and laboratory findings resulting from human herpesvirus-6 (HHV-6) in patients with mononucleosis-like illnesses not resulting from EpsteinBarr virus or cytomegalovirus. Am J Clin Pathol. 1990; 93(6):776-83. [PubMed: 2161178]

6. Smith JM, McDonald RA. Emerging viral infections in transplantation. Pediatr Transplant. 2006; 10(7):838-43. [PubMed: 17032433]

7. Yoshikawa T. Human herpesvirus-6 and -7 infections in transplantation. Pediatr Transplant. 2003; 7(1):11-7. [PubMed: 12581322]

8. Okuno T, et al. Seroepidemiology of human herpesvirus 6 infection in normal children and adults. $\mathbf{J}$ Clin Microbiol. 1989; 27(4):651-3. [PubMed: 2542358]

9. Santoro F, et al. CD46 is a cellular receptor for human herpesvirus 6. Cell. 1999; 99(7):817-27. [PubMed: 10619434]

10. Tang H, et al. CD134 is a cellular receptor specific for human herpesvirus-6B entry. Proc Natl Acad Sci U S A. 2013; 110(22):9096-9. [PubMed: 23674671]

11. He J, et al. Infection of primary human fetal astrocytes by human herpesvirus 6. J Virol. 1996; 70(2):1296-300. [PubMed: 8551599] 
12. Isaacson E, et al. Evidence of human herpesvirus 6 infection in 4 immunocompetent patients with encephalitis. Clin Infect Dis. 2005; 40(6):890-3. [PubMed: 15736026]

13. Soldan SS, et al. Association of human herpes virus 6 (HHV-6) with multiple sclerosis: increased IgM response to HHV-6 early antigen and detection of serum HHV-6 DNA. Nat Med. 1997; 3(12):1394-7. [PubMed: 9396611]

14. Boeckh M, et al. Emerging viral infections after hematopoietic cell transplantation. Pediatr Transplant. 2005; 9(Suppl 7):48-54. [PubMed: 16305617]

15. Dulery R, et al. Early human herpesvirus type 6 reactivation after allogeneic stem cell transplantation: a large-scale clinical study. Biol Blood Marrow Transplant. 2012; 18(7):1080-9. [PubMed: 22212513]

16. Tokimasa $\mathrm{S}$, et al. Ganciclovir is effective for prophylaxis and treatment of human herpesvirus-6 in allogeneic stem cell transplantation. Bone Marrow Transplant. 2002; 29(7):595-8. [PubMed: 11979309]

17. Leong HN, et al. The prevalence of chromosomally integrated human herpesvirus 6 genomes in the blood of UK blood donors. J Med Virol. 2007; 79(1):45-51. [PubMed: 17133548]

*18. Pellett PE, et al. Chromosomally integrated human herpesvirus 6: questions and answers. Rev Med Virol. 2012; 22(3):144-55. This report provides consensus response to the unexpected finding that HHV-6 integrates into the telomeric regions of host DNA. [PubMed: 22052666]

19. Black JB, et al. Growth properties of human herpesvirus-6 strain Z29. J Virol Methods. 1989; 26(2):133-45. [PubMed: 2693479]

20. Reymen D, et al. Antiviral activity of selected acyclic nucleoside analogues against human herpesvirus 6. Antiviral Res. 1995; 28(4):343-57. [PubMed: 8669893]

21. Ablashi DV, et al. Genomic polymorphism, growth properties, and immunologic variations in human herpesvirus-6 isolates. Virology. 1991; 184(2):545-52. [PubMed: 1653487]

*22. Prichard MN, et al. Synthesis and antiviral activities of methylenecyclopropane analogs with 6alkoxy and 6-alkylthio substitutions that exhibit broad-spectrum antiviral activity against human herpesviruses. Antimicrob Agents Chemother. 2013; 57(8):3518-27. New analogs cyclopropavir with superior activity in HHV-6A and HHV-6B are described with a good description of analytic methods. [PubMed: 23669381]

23. Manichanh C, et al. Susceptibility of human herpesvirus 6 to antiviral compounds by flow cytometry analysis. Cytometry. 2000; 40(2):135-40. [PubMed: 10805933]

24. De Clercq E, Naesens L. In search of effective anti-HHV-6 agents. J Clin Virol. 2006; 37(Suppl 1):S82-6. [PubMed: 17276375]

25. Gerdemann U, et al. Immunotherapeutic strategies to prevent and treat human herpesvirus 6 reactivation after allogeneic stem cell transplantation. Blood. 2013; 121(1):207-18. [PubMed: 23152545]

26. Yoshida M, et al. Comparison of antiviral compounds against human herpesvirus 6 and 7. Antiviral Res. 1998; 40(1-2):73-84. [PubMed: 9864048]

27. De Bolle L, et al. Role of the human herpesvirus 6 u69-encoded kinase in the phosphorylation of ganciclovir. Mol Pharmacol. 2002; 62(3):714-21. [PubMed: 12181449]

28. De Bolle L, et al. Human herpesvirus 6 infection arrests cord blood mononuclear cells in G(2) phase of the cell cycle. FEBS Lett. 2004; 560(1-3):25-9. [PubMed: 14987992]

29. De Bolle L, et al. Human herpesvirus 6 DNA polymerase: enzymatic parameters, sensitivity to ganciclovir and determination of the role of the A961V mutation in HHV-6 ganciclovir resistance. Antiviral Res. 2004; 64(1):17-25. [PubMed: 15451175]

30. Manichanh C, et al. Selection of the same mutation in the U69 protein kinase gene of human herpesvirus-6 after prolonged exposure to ganciclovir in vitro and in vivo. J Gen Virol. 2001; 82(Pt 11):2767-76. [PubMed: 11602788]

31. Ljungman $P$, et al. Effectiveness of ganciclovir against human herpesvirus- 6 excreted in saliva in stem cell transplant recipients. Bone Marrow Transplant. 2007; 39(8):497-9. [PubMed: 17334385]

32. Kogelnik AM, et al. Use of valganciclovir in patients with elevated antibody titers against Human Herpesvirus-6 (HHV-6) and Epstein-Barr Virus (EBV) who were experiencing central nervous system dysfunction including long-standing fatigue. J Clin Virol. 2006; 37(Suppl 1):S33-8. [PubMed: 17276366] 
33. Mechai F, et al. Human herpesvirus 6-associated retrobulbar optic neuritis in an HIV-infected patient: response to anti-herpesvirus therapy and long-term outcome. J Med Virol. 2007; 79(7): 931-4. [PubMed: 17516535]

34. Cacheux W, et al. HHV-6-related acute liver failure in two immunocompetent adults: favourable outcome after liver transplantation and/or ganciclovir therapy. J Intern Med. 2005; 258(6):573-8. [PubMed: 16313481]

35. Johnston RE, et al. HHV-6-related secondary graft failure following allogeneic bone marrow transplantation. Br J Haematol. 1999; 105(4):1041-3. [PubMed: 10554818]

36. Mookerjee BP, Vogelsang G. Human herpes virus-6 encephalitis after bone marrow transplantation: successful treatment with ganciclovir. Bone Marrow Transplant. 1997; 20(10): 905-6. [PubMed: 9404935]

37. Rieux C, et al. Human herpesvirus- 6 meningoencephalitis in a recipient of an unrelated allogeneic bone marrow transplantation. Transplantation. 1998; 65(10):1408-11. [PubMed: 9625030]

**38. Bounaadja L, et al. Analysis of HHV-6 mutations in solid organ transplant recipients at the onset of cytomegalovirus disease and following treatment with intravenous ganciclovir or oral valganciclovir. J Clin Virol. 2013; 58(1):279-82. Resistance to ganciclovir and valganciclovir can occur during immune suppression and this report characterized the rate of resistance of HHV-6B as low during a course of therapy. [PubMed: 23871165]

39. Isegawa $Y$, et al. Human herpesvirus 6 ganciclovir-resistant strain with amino acid substitutions associated with the death of an allogeneic stem cell transplant recipient. J Clin Virol. 2009; 44(1): 15-9. [PubMed: 18952495]

40. Shiraki K, et al. Phosphonoacetic acid inhibits replication of human herpesvirus-6. Antiviral Res. 1989; 12(5-6):311-8. [PubMed: 2561336]

41. Bethge W, et al. Successful treatment of human herpesvirus-6 encephalitis after bone marrow transplantation. Bone Marrow Transplant. 1999; 24(11):1245-8. [PubMed: 10642816]

42. Pohlmann C, et al. Cidofovir and foscarnet for treatment of human herpesvirus 6 encephalitis in a neutropenic stem cell transplant recipient. Clin Infect Dis. 2007; 44(12):e118-20. [PubMed: 17516391]

43. Bonnafous P, et al. Different mutations in the HHV-6 DNA polymerase gene accounting for resistance to foscarnet. Antivir Ther. 2007; 12(6):877-88. [PubMed: 17926642]

44. Breddemann A, et al. Case report: severe gastrointestinal inflammation and persistent HHV-6B infection in a paediatric cancer patient. Herpes. 2007; 14(2):41-4. [PubMed: 17939902]

45. Bonnafous $\mathrm{P}$, et al. Characterization of a cidofovir-resistant HHV-6 mutant obtained by in vitro selection. Antiviral Res. 2008; 77(3):237-40. [PubMed: 18241936]

46. Hostetler KY. Alkoxyalkyl prodrugs of acyclic nucleoside phosphonates enhance oral antiviral activity and reduce toxicity: current state of the art. Antiviral Res. 2009; 82(2):A84-98. [PubMed: 19425198]

47. Williams-Aziz SL, et al. Comparative activities of lipid esters of cidofovir and cyclic cidofovir against replication of herpesviruses in vitro. Antimicrob Agents Chemother. 2005; 49(9):3724-33. [PubMed: 16127046]

48. Bidanset DJ, et al. Oral activity of ether lipid ester prodrugs of cidofovir against experimental human cytomegalovirus infection. J Infect Dis. 2004; 190(3):499-503. [PubMed: 15243923]

49. Quenelle DC, et al. Oral treatment of cowpox and vaccinia virus infections in mice with ether lipid esters of cidofovir. Antimicrob Agents Chemother. 2004; 48(2):404-12. [PubMed: 14742188]

50. Marty FM, et al. CMX001 to prevent cytomegalovirus disease in hematopoietic-cell transplantation. N Engl J Med. 2013; 369(13):1227-36. [PubMed: 24066743]

51. Naesens L, et al. Antiviral activity of diverse classes of broad-acting agents and natural compounds in HHV-6-infected lymphoblasts. J Clin Virol. 2006; 37(Suppl 1):S69-75. [PubMed: 17276373]

52. De Bolle L, et al. Potent, selective and cell-mediated inhibition of human herpesvirus 6 at an early stage of viral replication by the non-nucleoside compound CMV423. Biochem Pharmacol. 2004; 67(2):325-36. [PubMed: 14698045]

53. Kern ER, et al. In vitro activity and mechanism of action of methylenecyclopropane analogs of nucleosides against herpesvirus replication. Antimicrob Agents Chemother. 2005; 49(3):1039-45. [PubMed: 15728900] 
54. James SH, et al. Cyclopropavir inhibits the normal function of the human cytomegalovirus UL97 kinase. Antimicrob Agents Chemother. 2011; 55(10):4682-91. [PubMed: 21788463]

55. Chou S, Bowlin TL. Cytomegalovirus UL97 mutations affecting cyclopropavir and ganciclovir susceptibility. Antimicrob Agents Chemother. 2011; 55(1):382-4. [PubMed: 21041510]

*56. Gentry BG, et al. Resistance of human cytomegalovirus to cyclopropavir maps to a base pair deletion in the open reading frame of UL97. Antimicrob Agents Chemother. 2013; 57(9):4343-8. This report describes a new mutation in CMV that phosphorylates cyclopropavir, a promising new drug that is highly active against HHV-6 and CMV. [PubMed: 23817384]

57. Prichard MN. Function of human cytomegalovirus UL97 kinase in viral infection and its inhibition by maribavir. Rev Med Virol. 2009; 19(4):215-29. [PubMed: 19434630]

**58. Komazin-Meredith G, et al. Human herpesvirus 6 U69 kinase phosphorylates the methylenecyclopropane nucleosides cyclopropavir, MBX 2168, and MBX 1616 to their monophosphates. Antimicrob Agents Chemother. 2013; 57(11):5760-2. The UL69 kinase form HHV-6 was shown for the first time to phosphorylate ganciclovir and cyclopropavir. [PubMed: 23979753]

59. Biron KK, et al. Potent and selective inhibition of human cytomegalovirus replication by 1263W94, a benzimidazole L-riboside with a unique mode of action. Antimicrob Agents Chemother. 2002; 46(8):2365-72. [PubMed: 12121906]

60. Prichard MN, et al. Benzimidazole analogs inhibit human herpesvirus 6. Antimicrob Agents Chemother. 2011; 55(5):2442-5. [PubMed: 21300829]

61. Underwood MR, et al. Inhibition of human cytomegalovirus DNA maturation by a benzimidazole ribonucleoside is mediated through the UL89 gene product. J Virol. 1998; 72(1):717-25. [PubMed: 9420278]

62. Efferth T, et al. Antiviral activity of artesunate towards wild-type, recombinant, and ganciclovirresistant human cytomegaloviruses. J Mol Med (Berl). 2002; 80(4):233-42. [PubMed: 11976732]

63. Kaptein SJ, et al. The anti-malaria drug artesunate inhibits replication of cytomegalovirus in vitro and in vivo. Antiviral Res. 2006; 69(2):60-9. [PubMed: 16325931]

64. Shapira MY, et al. Artesunate as a potent antiviral agent in a patient with late drug-resistant cytomegalovirus infection after hematopoietic stem cell transplantation. Clin Infect Dis. 2008; 46(9):1455-7. [PubMed: 18419454]

65. Wolf DG, et al. Human cytomegalovirus kinetics following institution of artesunate after hematopoietic stem cell transplantation. Antiviral Res. 2011; 90(3):183-6. [PubMed: 21443904]

66. Milbradt J, et al. Sensitivity of human herpesvirus 6 and other human herpesviruses to the broadspectrum antiinfective drug artesunate. J Clin Virol. 2009; 46(1):24-8. [PubMed: 19501020]

*67. Hakacova N, et al. First therapeutic use of Artesunate in treatment of human herpesvirus 6B myocarditis in a child. J Clin Virol. 2013; 57(2):157-60. For the first time artesunate was used for compassionate therapy for HHV-6 myocardits rather than ganciclovir, foscarget or cidofovir. [PubMed: 23473961]

68. Naesens L, et al. Antiviral properties of new arylsulfone derivatives with activity against human betaherpesviruses. Antiviral Res. 2006; 72(1):60-7. [PubMed: 16650489] 


\section{Highlights}

- The virus is inhibited in cell culture by the approved therapies cidofovir and foscarnet

- The investigational drug, brincidofovir is effective in cell culture

- Interest in the development of specific inhibitors is subdued because of a perceived lack of a treatable disease 

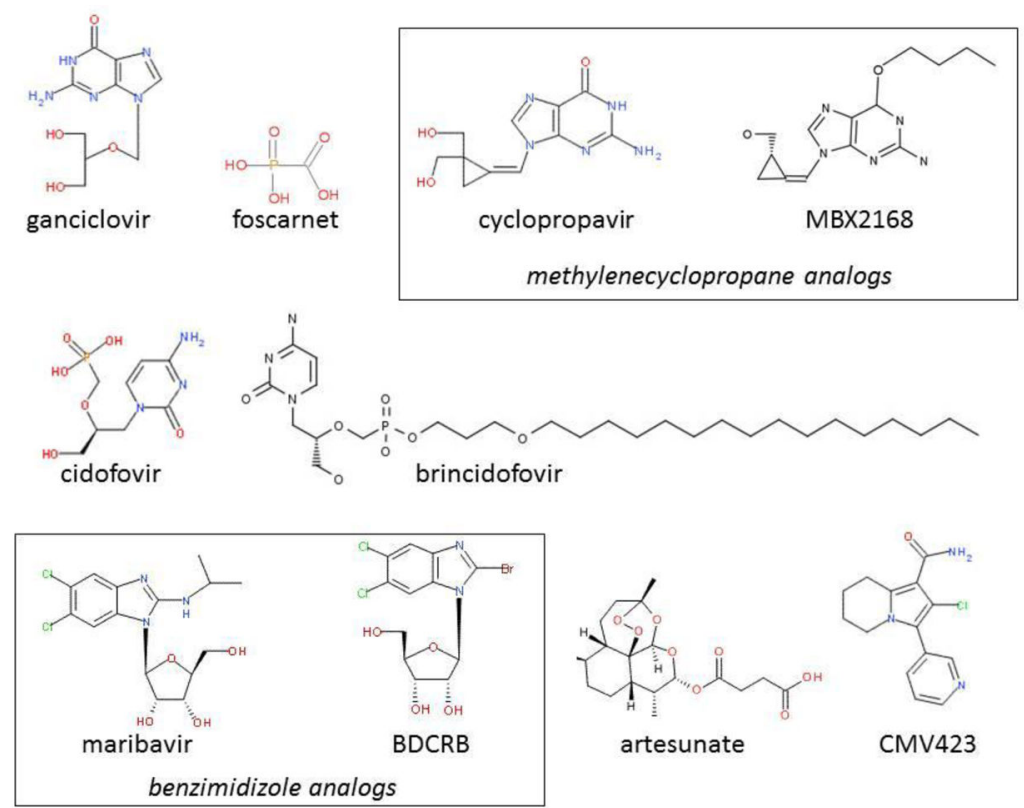

Figure 1.

Structures for selected compounds with antiviral activity. Classes of specific compounds are designated in boxes. The structure for D BDCRB is shown although both the L and D isomers are discussed. 
